\title{
THE COHOMOLOGY OF COMPACT ABELIAN GROUPS
}

\author{
BY KARL HEINRICH HOFMANN ${ }^{1}$ AND PAUL S. MOSTERT ${ }^{2}$
}

Communicated by A. Borel, March 25, 1968

There are at least two (if not more) cohomology theories for a compact group $G$. The first is the space cohomology of the topological space underlying $G$ based on Cech cochains, say; the second is an extension of the algebraic cohomology of finite groups based on, say, the Cech cochains with compact support on the classifying space of $G$. This note is concerned with the second cohomology for compact abelian groups. However, for the sake of completeness we recall the main results of the first theory [2].

TheOREM 1. If $G$ is a compact abelian group, $R$ a commutative ring with identity, then the Cech cohomology $\check{H}(G, R)$ is a graded commutative Hopf algebra over $R$ and is naturally isomorphic to the Hopf algebra $R \otimes C(G, Z) \otimes \Lambda\left(G_{0}\right)^{\wedge}$, where $\Lambda X$ is the integral exterior algebra over the group $X$ in degree 1 with its natural Hopf algebra structure, and where $C(G, Z)$ is the Hopf algebra of all continuous functions from $G$ into the discrete ring $\boldsymbol{Z}$.

CoROLlaRY 2. If $G$ is a compact connected abelian group and $R a$ commutative ring with identity, then there is a natural isomorphism of commutative Hopf algebras $\hat{H}(G, R) \cong R \otimes \Lambda \hat{G}$.

Definition 3. Suppose that $E^{1}(G) \rightarrow \cdots E^{n}(G) \rightarrow \cdots E(G)$ $=E^{\infty}(G)$ is a sequence of spaces and injective maps such that (i) $E^{n}(G)$ is compact for $n<\infty$, (ii) $\check{H}^{i}\left(E^{n}(G), \boldsymbol{Z}\right)=0$ for $0<i<n ; n \leqq \infty$, and that (iii) $G$ acts freely on all spaces. Then $B^{n}(G)$ is called a classifying space up to $n$ (resp. just a classifying space for $n=\infty$ ), and for an arbitrary $R$-module $A$ the graded $R$-module proj $\lim _{n} \breve{H}\left(B^{n}(G), A\right)$ is independent of the particular choice of a system of classifying spaces. If $A=R$ then the ring structure on $\breve{H}\left(B^{n}(G), R\right)$ gives the limit a graded $R$-algebra structure in a natural fashion. The limit will be called $h(G, A)$.

Functorial constructions for classifying spaces have been given by Milnor, Dold and Lashof, Rothenberg and Steenrod. There is a natural morphism $\check{H}(B(G), A) \rightarrow h(G, A)$, but it is not entirely clear whether it is an isomorphism for all compact groups.

\footnotetext{
1 Fellow of the Alfred P. Sloan Foundation.

2 Holder of a National Science Foundation Senior Postdoctoral Fellowship.
} 
Proposition 4. Let $G$ be a compact connected abelian group and $R$ any commutative ring with identity. Then there is an isomorphism of graded commutative Hopf algebras $h(G, R) \cong R \otimes P \hat{G}$, where $P X$ is the integral symmetric algebra over the group $X$ in degree 2 with its standard Hopf algebra structure. In particular, $h(G, Z)=P \hat{G}$.

Note. Compare Corollary 2, Proposition 4 and [5, Theorem $\mathrm{H}^{*}$ ].

The more general case of a not necessarily connected, compact abelian group is considerably more complicated than one might expect after Proposition 4 drawing from analogy with Theorem 1 . In order to make a first observation, we remark that for any compact abelian group the exterior algebra $\Lambda G$ can be defined so that it has the familiar properties of the discrete case. There is a natural isomorphism $\bigwedge G \rightarrow \Lambda G / G_{0}$.

THEOREM 5. Let $G$ be a compact abelian group and $R$ any commutative group (resp. ring with identity). Then there are natural injections of graded abelian groups (resp. R-algebras)

$$
\begin{aligned}
& \tau_{G, R}: R \otimes P \hat{G} \rightarrow h(G, R), \\
& \rho_{G, R}: \operatorname{Hom}(\Lambda G, R) \rightarrow h(G, R) .
\end{aligned}
$$

(In (2) and in (3) below, $\operatorname{Hom}(\Lambda G, R)$ denotes the group of all continuous group morphisms into the discrete group $R$; the gradation is the obvious one.)

Consequently, there is a natural morphism of graded abelian groups (resp. R-algebras)

$$
\omega_{G, R}: R \otimes P \hat{G} \otimes \operatorname{Hom}(\Lambda G, R) \rightarrow h(G, R) .
$$

The group morphism $\omega_{G, R}^{i}$ is bijective for $i=0,1,2$ if $R$ is a principal ideal domain with zero characteristic.

Note that after Theorem 5 there is a natural $R \otimes P \hat{G}$-module structure on $h(G, R)$ via (1), if $R$ is a ring.

Definition 6. Let $\phi: A \rightarrow B$ be a morphism of $R$-modules over some commutative ring with identity, and $P_{R} B$ the symmetric $R$-algebra over $B$ in degree 2 . Let $E_{2}(\phi)$ denote the differential bigraded algebra $P_{R} B \otimes_{R} \Lambda_{R} A$ with the differential $d_{\phi}$ of bidegree $(2,-1)$ characterized by $d_{\phi}(x \otimes 1)=0$ and $d_{\phi}(1 \otimes a)=\phi(a) \otimes 1$ for $a \in A$. Let $E_{3}(\phi)$ denote the bigraded algebra derived from $E_{2}(\phi)$ by passing to cohomology.

Definition 7. A standard resolution of a finite abelian group $G$ is an exact sequence

$$
0 \rightarrow F \stackrel{f}{\rightarrow} F \stackrel{\pi}{\rightarrow} G \rightarrow 0
$$


of abelian groups in which $f=f_{1} \oplus \cdots \oplus f_{n}$ so that $\operatorname{dom} f_{i}=\operatorname{codom} f_{i}$ $\cong Z$, and $f_{i} x=z_{i} x$ with natural numbers $z_{i}$ satisfying $z_{i} \mid z_{i+1}$, $i=1, \cdots, n-1$.

Note that every finite abelian group admits such a resolution in an essentially unique way.

Lemma 8. Let $G$ be a compact abelian Lie group and let $R$ be a commutative ring with identity. Then there is an isomorphism of graded commutative rings

$$
h(G, R) \cong P\left(G_{0}\right)^{\wedge} \otimes E_{3}(\operatorname{Hom}(f, R)),
$$

where $0 \rightarrow F \stackrel{f}{\rightarrow} F \rightarrow \rightarrow G / G_{0} \rightarrow 0$ is a standard resolution of $G / G_{0}$ and where the $R$-module action on the right is the obvious one defined by the fact that $E_{3}$ is an $R$-algebra.

In point of fact, there is a natural isomorphism $E_{3}(\operatorname{Hom}(f, R))$ $\cong H\left(G / G_{0}, R\right)$, where $H$ denotes the algebraic cohomology [3]. The properties of the spectral algebras $E_{r}(\operatorname{Hom}(f, R)), r=2,3$ are studied extensively by the authors in a forthcoming paper [3].

Since $h(-, R)$ transforms projective limits into direct limits, Lemma 8 makes $h(G, R)$ amenable to computation, at least in principle. However, explicit results are not easy to obtain.

CoRollary 9. If $0 \rightarrow G_{0} \stackrel{i}{\rightarrow} G \stackrel{\pi}{\rightarrow} G / G_{0} \rightarrow 0$ is the exact sequence defined by the identity component $G_{0}$ of a compact abelian group $G$, then $h(\pi, R): h\left(G / G_{0}, R\right) \rightarrow h(G, R)$ is injective and $h(i, R): h(G, R) \rightarrow h\left(G_{0}, R\right)$ is surjective.

Despite Lemma 9, in general we do not have $h(G, R) \cong h\left(G_{0}, R\right)$ $\otimes h\left(G / G_{0}, R\right)$, not even for $\operatorname{dim} G=1, G_{0}=\hat{Q}, R=\boldsymbol{Z}$. However, if $R$ is a field, then the situation is better:

TheOREM 10. Let $G$ be a compact abelian group and $R$ a field with prime field $K$. Then $\omega_{G, R}$ is an isomorphism and there is a natural isomorphism of graded commutative Hopf algebras

(4) $\quad R \otimes P\left(G_{0}\right)^{\wedge} \otimes P \operatorname{Tor}(\hat{G}, K) \otimes \Lambda$ Tor $(\hat{G}, K) \rightarrow h(G, R)$.

CoRollaRY 11. If $G$ is a compact abelian group, then $h(G, R)$ $\cong h\left(G_{0}, R\right) \cong P_{R} \operatorname{Hom}(R, G)^{*} \cong P_{R}(R \otimes \hat{G})$, where the asterisk denotes the dual of a vector space.

Corollary 12. Let $G$ be a compact abelian group. Then there is a natural isomorphism of Hopf algebras

$$
\begin{aligned}
h(G, G F(p)) \cong G F(p) \otimes P\left(G_{0}\right)^{\wedge} \otimes P \hat{G} / p \hat{G} \otimes \Lambda \hat{G} / p \hat{G} & \\
& =G F(p) \otimes P\left(G_{0}\right)^{\wedge} \otimes E_{2}(e),
\end{aligned}
$$

where $e$ is the identity map of $\hat{G} / p \hat{G}$. The differential 


$$
G F(p) \otimes P\left(G_{0}\right)^{\wedge} \otimes d_{e}
$$

(see Definition 6) corresponds to the Bockstein derivation of $h(G, G F(p)$ ) under this isomorphism. Hence there is a natural exact sequence

$$
\begin{aligned}
0 \rightarrow p h^{+}(G, \boldsymbol{Z}) \cap p \text {-socle } h^{+}(G, \boldsymbol{Z}) \rightarrow p \text {-socle } h^{+}(G, \boldsymbol{Z}) \\
\rightarrow \operatorname{im} G F(p) \otimes P\left(G_{0}\right)^{\wedge} \otimes d_{c} \rightarrow 0,
\end{aligned}
$$

where $h^{+}$designates the ideal generated by the elements of positive degree.

As far as $h(G, Z)$ in general is concerned, Lemma 8 and the general theory of the spectral algebras $E_{r}$ enable us to produce in a canonical fashion a minimal subgroup of $h(G, \boldsymbol{Z})$ which generates $h(G, \boldsymbol{Z})$ as a $P \hat{G}$-module (and thus almost as a ring):

THEOREM 13. Let $G$ be a compact abelian group and let $b^{i}: h^{i}(G, R / Z)$ $\rightarrow h^{i+1}(G, \boldsymbol{Z}), i=0,1, \cdots$, be the connecting morphism in the long exact sequence arising from the coefficient sequence $0 \rightarrow \boldsymbol{Z} \rightarrow \boldsymbol{R} \rightarrow \boldsymbol{R} / \boldsymbol{Z} \rightarrow \mathbf{0}$. (If $G$ is totally disconnected, then $b^{i}$ is an isomorphism.) If $p$ is the morphism given in (2) of Theorem 5 , then the graded subgroup $M=Z$ $\oplus \operatorname{im} b \rho_{G, R / Z}$ is a minimal subgroup such that $h(G, Z)=(P \hat{G}) \cdot M$ with the $P \hat{G}$-module structure of $h(G, Z)$ afforded by (1) of Theorem 5 . The subgroup $M+h^{2}(G, Z)$ generates the ring $h(G, Z)$. (Recall $h^{2}(G, Z) \cong \hat{G}$.) As a graded abelian group, $M$ is isomorphic to $(\Lambda G)^{\wedge}$ under $b_{\rho_{G}, R / z}$ with a shift in degree. As a $P \hat{G}$-module, $h(G, Z)$ is torsion free.

We remind the reader that a totally disconnected compact abelian group $G$ is a direct product of its $p$-primary components $G(p)$ such that $G(p)$ is a maximal pro- $p$-subgroup. One of the sample corollaries of the theory is

Proposition 12. A compact abelian group $G$ has a compact classifying space if and only if it is totally disconnected and $G(p)$ is a product of $a$ (possibly empty) collection of p-adic groups for each prime $p$.

\section{REFERENCES}

1. A. Borel, Sur la cohomologie des espaces fibrés principaux et des espaces homogènes de groupes de Lie compacts, Ann. of Math. 57 (1953), 115-207.

2. K. H. Hofmann, Categories with convergence, exponential functors, and the cohomology of compact abelian groups, Math. Z. 104 (1968), 106-144.

3. K. H. Hofmann and P. S. Mostert, The cohomology of finite and compact abelian groups (to appear).

4. D. Husemoller, Fibre bundles, McGraw-Hill, New York, 1966.

5. T. Petrie, The Eilenberg-Moore, Rothenberg-Steenrod spectral sequence for $K$ theory, Proc. Amer. Math. Soc. 19 (1968), 193-194.

TUlane University and

The Institute for Advanced Study 